\title{
The role of dendritic cells in the pathogenesis of Lyme disease
}

\author{
ANNA MONIUSZKO ${ }^{1}$, PAWEŁ PENZA', PIOTR CZUPRYNA ${ }^{l}$, SEAWOMIR PANCEWICZ1, \\ JOANNA ZAJKOWSKA ${ }^{1}$
}

${ }^{2}$ Department of Infectious Diseases and Neuroinfections, Medical University of Bialystok, Bialystok, Poland

${ }^{1}$ Department of Internal Medicine, Provincial Hospital, Zambrow, Poland

\begin{abstract}
Pathophysiology of Lyme disease (LD) is complicated and depends on various factors such as individual presentation of antigens, bacterial genotype, early proper diagnosis and response to treatment. Borrelia burgdorferi localised in the skin, is covered by a coat made of saliva proteins Salp, presents mostly OspC and blocks the complement system by using CRASP. Dendritic cells (DCs) act as the first line of specific immunological response, which influences the disease.

The type of DCs, level of maturity, proportion of DCs and T lymphocytes influence the level and type of the immunological response. Lyme disease presents mainly immunological response on Th1 lymphocytes pathway, which is proven by the synthesis of interleukin 12 (IL-12) and interferon $\gamma(I F N-\gamma)$ in the early phase of inflammation. In a later phase, Th2 response is activated with a secondary increase in specific cytokines IL-10 or IL-4. Therefore, DCs play one of the main functions in the pathogenesis of the disease in relation to the synthesis and stimulation of other cells to release inflammatory mediators. Bacterial ability to invade and disperse in the organism is additionally supported by their vector. Ticks, via local immunosuppressive activity, inhibit early antibacterial mechanisms connected with DCs and therefore favor the infection.

This paper describes the role of DCs as important factors in the pathogenesis of LD in both non-specific and specific immunological response.

We conclude, that considering a complex role of DCs in defense mechanisms and initiation process of direct immunological response, their role should be accounted in vaccination against-Borrelia burgdorferi.
\end{abstract}

Key words: dendritic cells, Lyme disease, Borrelia burgdorferi.

(Centr Eur J Immunol 2013; 38 (4): 569-577)

\section{Introduction}

Borreliosis is a zoonotic infectious disease caused by Borrelia burgdorferi (B. burgdorferi) sensu lato: garinii, afzelii and sensu stricto. It occurs in Northern America, Europe and Asia. Borrelia burgdorferi sensu stricto transmitted by Ixodes scapularis in the USA, whereas B. garinii and $B$. afzelii transmitted by Ixodes ricinus in Europe. The disease may involve the central nervous system, joints, muscles, skin and cardiovascular system, but the most often it occurs as erythema migrans (EM) (Fig. 1) [1].

Skin separates the human body from the environment, hence it plays a crucial role in the protection against pathogenic factors. For both, the tick and the spirochete, it is of paramount importance to inhibit the host innate immune response, the first line of host defense. Borrelia is transmitted to humans by a bite of the infected tick. At least 48-72 hours' period of the tick contact with the skin is required for the bacteria for effective transmission. Primary infection is localized in the skin, which constitutes an important element of the immune system [1].

Importantly, immunosuppressive influence of tick saliva may result in more efficient transmission of several tick-borne pathogens. There are several cells involved in the immunological response, altogether known as skin-associated lymphoid tissue (SALT).

It has been proven that dendritic cells (DCs) play an important role in the regulation of the innate and adaptive immunological response [2-4].

Innate response is based on the production of cytokines, which stimulate the immune system. A crucial part of the innate immune response against invading microorganisms are composed of:

- the complement cascade (B. burgdorferi utilizes complement regulating-acquiring surface proteins - CRASP),

Correspondence: Anna Moniuszko, Department of Infectious Diseases and Neuroinfections, Medical University in Bialystok, Żurawia 14, 15-540 Bialystok, Poland, tel. +48 8574095 14, fax +48 8574095 15, e-mail: annamoniuszko@op.pl 


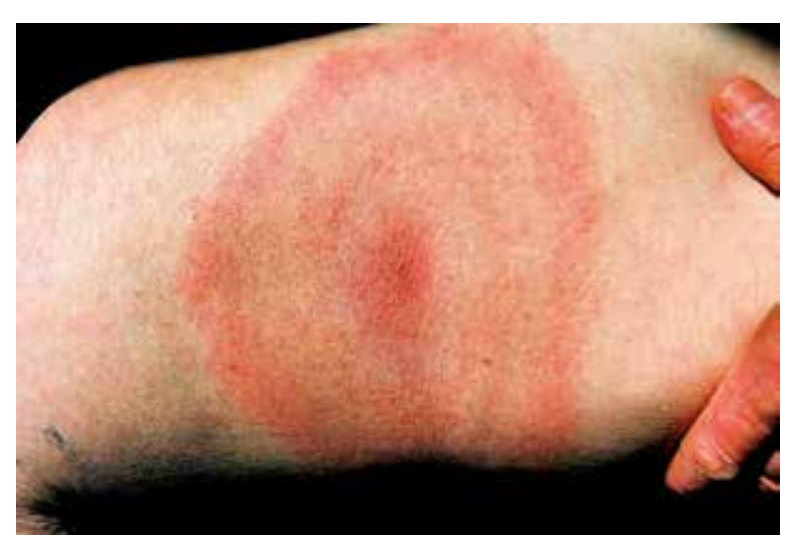

Fig. 1. Erythema migrans

and Osp E/F related proteins to bind factor $\mathrm{H}$ or FHL protein and consequently inhibit,

- the family of antimicrobial peptides - a form of host defense found in both plants and animals, which possesses bactericidal capacities. Production of antimicrobial peptides at the site of infection, for instance, the skin, also results in chemotaxis of leukocytes. Borrelia burgdorferi is able to induce the production of several antimicrobial peptides, including human $\beta$-defensin- 2 and cathelicidin LL-37, in human resident skin cells (fibroblasts and keratinocytes),

- mast cells,

- macrophages,

- NK cells,

- neutrophils and DCs,

- fibroblasts and keratinocytes [3].

Adaptive response is based on the location and transformation of antigens before their presentation to the lymphocytes. Moreover, they may directly activate B lymphocytes.

Pathogens, which enter the skin, activate DCs of the proper layer of the skin, may be captured by the monocytes of the peripheral blood or may directly migrate to the lymph nodes and be presented to $\mathrm{T}$ and $\mathrm{B}$ lymphocytes [5]. Skin constitutes the area of an early and active defense in the tick-borne diseases, including Lyme borreliosis. Tick adaptation to pain-free preying on the host and initiating local immunosuppression via saliva components allow deposition of B. burgdorferi spirochaetes in the skin. Due to unique properties, such as the ability to inactivate complement cascade with the CRASP proteins, the presence of the proper lipoproteins $(\mathrm{OspC})$, adhesion to integrins, proteoglycans, decorin, plasmin bind, serine protease, the activation of host's metalloproteinases penetration and destruction of the extracellular matrix, interaction with the mesothelial cells, platelets and collagen is possible, however further elimination of the bacteria does not take place.

In humoral immunity, antigen-presenting cells engulf and break down B. burgdorferi [6]. Antigens are then brought to the outside surface of the antigen-presenting cells and presented in conjunction with class II MHC proteins. The helper T-cells recognize the antigen presented in this way and release cytokines, proteins that signal B-cells to take further action. B-cells that are stimulated in this way develop into plasma cells, which secrete antibodies specific to the recognized antigen.

Antibodies are proteins present in the circulation, and on the surface of B-cells [7]. They can destroy the bacteria from which the antigen came. Destruction occurs either directly, or by tagging the organism, which will then be more easily recognized and targeted by phagocytes and complement proteins. Some of the stimulated B-cells go on to become memory cells, which are able to mount an even faster response if the antigen is encountered for the second time.

Borrelia burgdorferi move with the potent high motility, what enables fast dissemination and settlement in other tissues $[8,9]$. Dendritic cells are the first-line defense in the skin and influence the course of the infection. Proper immune response is one of the most important factors of the self-limitation of infection. The most common, early symptom of the skin lesion is EM or borrelial lymphocytoma (BL) $[1,10,11]$. The chronic stadium of the disease, which occurs a few years after the infection, is acrodermatitis chronica atrophicans (ACA). Dendritic cells play a role in all the stages of skin LD.

\section{Mechanisms facilitating Borrelia burgdorferi infection}

The most important effect of the activity of tick's saliva is inhibition of T-cells proliferation and inhibition of Th1 cytokines release, such as interferon $\gamma($ IFN- $\gamma$ ) and interleukin 2 (IL-2). During the presence on a host, the tick releases substances, which facilitate survival, e.g. anticoagulants, vasodilators (inhibitors releasing antimicrobial peptides) and immunosuppressive compounds. Dendritic cells function is influenced by Salp15 $[9,12]$. This is a protein of tick's salivary glands, which indirectly inhibits T lymphocytes activation [13].

Salp15 interacts mainly with DC-SIGN receptor on DCs, what lead to the reduction of proinflammatory cytokines secretion (IL-12, IL-6, TNF- $\alpha$ ), essential in $\mathrm{T}$ lymphocytes activation in lymph nodes, and directly binds CD4 receptors of the lymphocytes inhibiting them in the area of inflammation. Both mechanisms are complementary to each other and cause the decrease in the number of effector lymphocytes and the development of improper adaptive immunological response. Similar inhibiting properties of Salp15 were observed in the secretion of proinflammatory cytokines (IL-12p70, IL-6, TNF- $\alpha$ ) released by DCs as a response to LPS antigen. Other immunomodulators in the tick's saliva are: PGE2, 36kDa protein, IL-2 binding protein, sialostatin L (Sialol), which 
inhibits cysteine protease. This inhibitor has high affinity towards cathepsin L and partial cathepsin S [14].

The bond of cathepsin S inside the DCs leads to the disturbances and difficulties in the final processing of antigens and the production of MHC II complexes. Hence, Sialol inhibits pathogen induced DCs maturation and leads to the inhibition of antigen specific T-lymphocytes proliferation. In animal models, Vesely et al. observed that the presence of a pathogen can overcome the Th1-inhibitory effects of tick feeding on the host [15]. Hence, local immunosuppression due to the activity of the tick facilitates transmission and the development of infection with B. burgdorferi.

Another interesting observation was performed by Skallova et al., who observed that exposure of DCs to tick saliva in vitro resulted in impaired maturation, upon CD40 or TLR9, TLR3 and TLR7 ligation, as well as reduced Ag presentation capacity. Administration of tick saliva in vivo significantly inhibited maturation and early migration of DCs from skin to lymph nodes, and decreased the capacity of lymph node DCs to present soluble antigen (Ag) to specific T cells. Moreover, saliva-exposed DCs failed to induce efficient Th1 and Th17 polarization and promoted development of Th2 responses. These observations revealed a complex inhibitory effect exerted by tick saliva on DCs function. Given the role of DCs as the crucial stage of adaptive immune responses, alteration of their function might represent a major mechanism of tick-mediated immune evasion [16].

\section{Dendritic cells}

Antigen presentation is a key moment of the initiation of the immune response. Insufficient ability of direct antigen binding and therefore self-activation of T lymphocytes contributes to the fact that recognition of foreign antigens requires close cooperation with antigen presenting cells (APC).

Dendritic cells are a group of highly specialized cells transforming and presenting foreign antigens (APCs) [7, 17]. They are found in many locations of potential contact with the pathogen (skin, mucous membranes) and in other tissues of the body (synovial fluid, CNS, blood, peripheral lymph nodes). These cells are immature, inactive with the ability to phagocytosis of the bacteria. After the phagocytosis of the pathogen, DCs are activated, they mature and migrate to the peripheral lymphatic organs. In the meantime, they transform proteins of the antigen, therefore increase their immunogenic potential. Moreover, they synthesize glycoproteins of the major histocompatibility complex (MHC II). Such complexes are further exposed on the cell surface and are presented to lymphocytes [18].

Major histocompatibility complex molecules assemble within the cell where they associate with short peptide fragments derived either from proteins being made by the cell (MHC class I molecules bind to peptides derived from proteins being synthesized within the cell) or proteins that have been internalized by the cell through phagocytosis or pinocytosis (MHC class II molecules bind to peptides derived from proteins made external to the cell).

One particular type of antigen presentation is cross-presentation: extracellular antigen is not classically presented in the context of MHC-II but is instead shunted into the MHC-I presentation pathway [19, 20]. CD8+ T cells can thus be activated by antigens taken up from the extracellular space and then differentiate into cytotoxic T cells. This mechanism is thought to be of major importance for the recognition of viral or bacterial antigens when DCs are not directly infected [21].

Dendritic cells not only influence the type of T-cell response, but also participate in the activation and recruitment of the immature DC, NK, macrophages, granulocytes and B-lymphocytes. A large amount of DCs located in the skin constitute a vital primary specialized system of defense of the immune system against $B$. burgdorferi, therefore play a key role in the pathogenesis of LD (Fig. 2).

Dendritic cells are divided classically into several subgroups:

- sentinel cells, which are Langerhans cells (CD1a+) of the epithelium and mucous membrane,

- so-called myeloid dendritic cells (CD11c+; MDCs) located in many tissues and places of potential contact with the antigens in the peripheral lymphatic organs and blood,

- so-called plasmacytoid DCs (CD11c; pDCs) present in the blood and peripheral lymphatic organs in the areas abundant in T lymphocytes [7, 22].

Myeloid DCs have a high myeloid antigen level, possess high ability to phagocytosis, but they lack the ability to reproduce. Plasmacytoid DCs have a low myeloid antigen level, low ability to phagocytosis, however they produce a large amount of IFN in a response to infection. Myeloid DCs absorb penetrating pathogens, what activates the process of their maturation. During this process they transform and migrate to secondary lymphatic organs in order to present antigens, together with the antigen of the MHC, to T lymphocytes. Depending on the maturation conditions MDC induce Th1 (key lymphocytes for the cell type response with Tc lymphocytes) or Th2 (role in the humoral response with B lymphocytes) - dependent immunological response. However, the main function of $\mathrm{pDCs}$ is the production of type I IFN in a response to infection. It has also been proven that these cells become APC and induce Th2-dependent response in the presence of IL-3 in the process of maturation [22].

However recently, a number of studies have demonstrated that MDCs, or conventional/classical DCs, may be further subdivided into several subgroups. In mice, the CD8+ and the CD11b+ lymphoid resident DCs, as well as the CD103+ and CD11b+ migratory DCs were subdivided. Homologous DC populations have also been identified in 

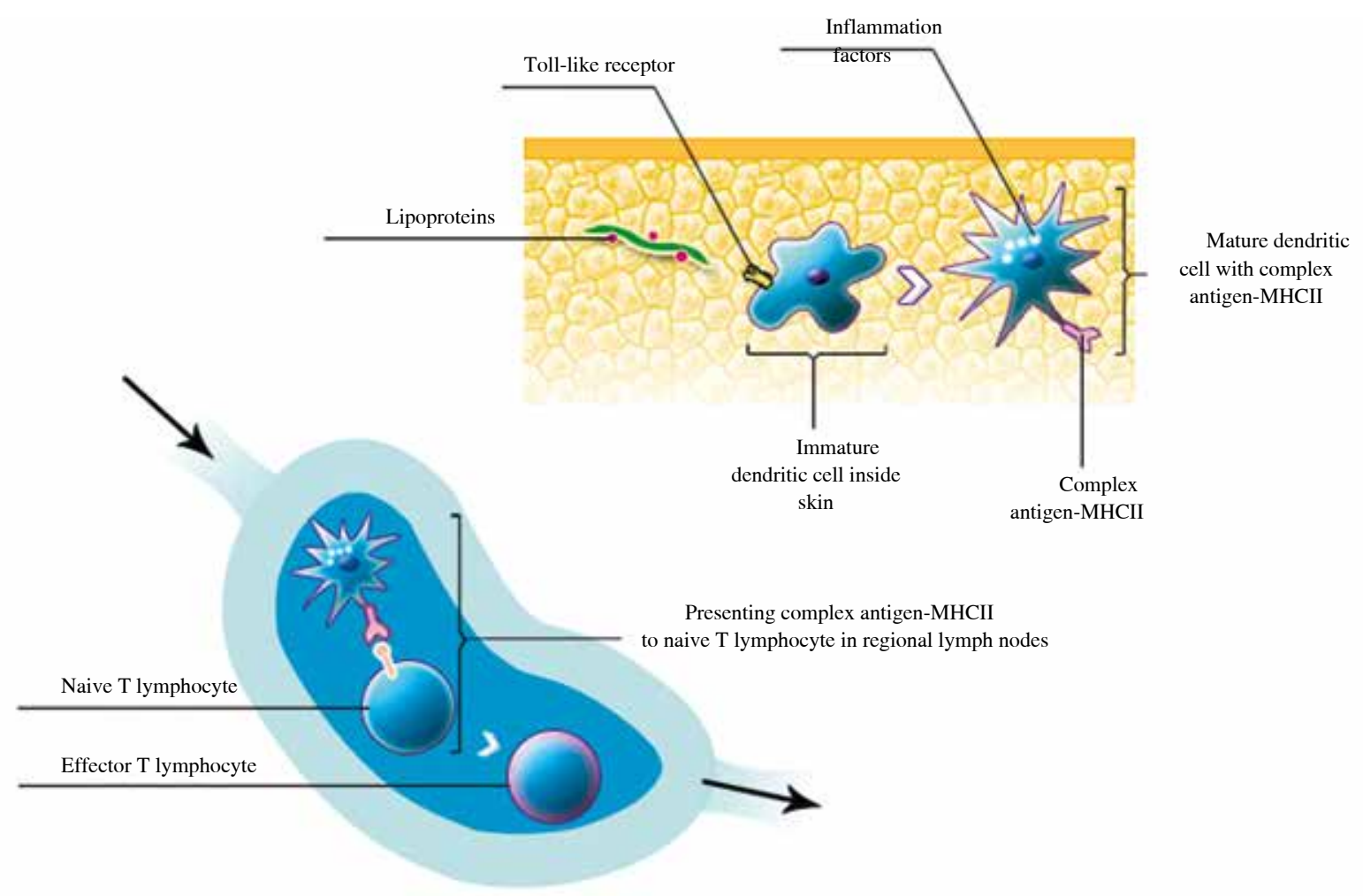

Fig. 2. Role of dendritic cells in immunity

humans with BDCA3+ (CD141) DCs sharing homology with CD8+ DCs and BDCA1 (CD1c) DCs being similar to $\mathrm{CD} 11 \mathrm{~b}+\mathrm{DCs}$ [23].

Bachem et al. showed that CD141+ DCs are the only cells in human blood that express the chemokine receptor XCR1 and respond to the specific ligand XCL1 by $\mathrm{Ca}^{2+}$ mobilization and potent chemotaxis. More importantly, they demonstrated that CD141+ DCs excel in cross-presentation of a soluble or cell-associated antigen to CD8+ T cells when directly compared with CD1c+ DCs, CD16+ DCs, and pDCs from the same donors. Both in their functional XCR1 expression and their effective processing and presentation of exogenous antigen in the context of MHC class I, human CD141+ DCs correspond to mouse CD8+ DCs, a subset known for superior antigen cross-presentation in vivo [24].

Recent evidence shows that mouse non-lymphoid tissue CD103+ DCs and human blood DC Ag 3+ DCs share similarities with $\mathrm{CD} 8 \mathrm{a}+\mathrm{DCs}$. Contreras et al. demonstrated that the minor sheep CD26+ skin lymph DC subset shares significant transcriptomic similarities with mouse CD8a+ and human blood DC Ag 3+ DCs. This enabled identification of a common set of phenotypic characteristics for CD8a-like DCs in the mammalian species. Compared to CD262 DCs, the sheep CD26+ DCs show potent stimulation of allogeneic naive CD8+ T cells with high selective induction of the Ifng and Il22 genes. It also showed dominant efficacy in activating specific CD8+ T cells against exogenous sol- uble Ag; and selective expression of functional pathways associated with high capacity for Ag cross-presentation. Their results unraveled a unifying definition of the CD8a+like DCs across mammalian species and identification of molecular candidates that could be used for the design of vaccines applying to mammals in general [25].

In 2012, Haniffa et al. identified a CD141hi DC present in human interstitial dermis, liver, and lungs that was distinct from the majority of CD1c+ and CD14+ tissue DCs and superior at cross-presenting soluble antigens. Cutaneous CD141+ DCs were closely related to blood CD141+ DCs, and migratory counterparts were found among skin-draining lymph node DCs. Comparative transcriptomic analysis with mouse showed tissue DC subsets to be conserved between species and permitted close alignment of human and mouse DC subsets. Results of their studies informed the rational design of targeted immunotherapies and facilitated translation of mouse functional DC biology to the human setting [26].

\section{Dendritic cells and Borrelia burgdorferi invasion}

Histological examination of EM is characterized by perivascular influx of lymphocytes, DCs, macrophages and plasmatic cells [27]. The typical feature of EM is a low number of neutrophils, which is quite atypical in 
Table 1.

\begin{tabular}{|c|c|c|c|}
\hline Cell type & Cell subset & Characteristics & Main functions \\
\hline $\begin{array}{l}\text { dendritic cells (DCs) } \\
\text { located in human skin }\end{array}$ & $\begin{array}{l}\text { Langerhans cells (LCs) } \\
\text { ("sentinel" DC) }\end{array}$ & $\begin{array}{c}\text { Marker: CD1a+ } \\
\text { location (skin, mucosa surface) } \\
\text { low phagocytic capacity than mDCs }\end{array}$ & $\begin{array}{l}\text { recognition of pathogens by PRRs, } \\
\text { antigen processing and presentation, } \\
\text { mediate preferentially cytotoxic T cell } \\
\text { response }\end{array}$ \\
\hline \multirow[t]{2}{*}{$\begin{array}{l}\text { dendritic cells (DCs) in } \\
\text { blood }\end{array}$} & myeloid DC (mDCs) & $\begin{array}{c}\text { high levels of myeloid antigens (CD11c, } \\
\text { CD13, CD33) } \\
\text { marker: CD11c } \\
\text { growth factor: GM-CSF } \\
\text { do not proliferate } \\
\text { high phagocytic capacity } \\
\text { are found in many tissues at sites of } \\
\text { antigen delivery in secondary lymphoid } \\
\text { organs and in blood }\end{array}$ & $\begin{array}{l}\text { recognition of pathogens by PRRs, } \\
\text { antigen processing and presentation, } \\
\text { production of inflammatory cytokines } \\
\text { and chemokines }\end{array}$ \\
\hline & plasmacytoid DC (pDCs) & $\begin{array}{c}\text { low levels of myeloid antigens } \\
\text { marker: IL-3R } \alpha \text { (CD123, or } \alpha \text {-chain of } \\
\text { IL-3 receptor) } \\
\text { growth factor: IL-3 } \\
\text { proliferate in response to IL-3 } \\
\text { low phagocytic capacity } \\
\text { have been identified in blood and in } \\
\text { T-cell-rich areas of secondary lymphoid } \\
\text { organs }\end{array}$ & $\begin{array}{l}\text { recognition of pathogens by PRRs, } \\
\text { antigen } \\
\text { processing and presentation, type I IFN } \\
\text { production, production of inflammatory } \\
\text { cytokines and chemokines }\end{array}$ \\
\hline
\end{tabular}

comparison with other bacterial infections. It should be emphasized that DCs also secrete IL- 8 which, as in the case of LPS presence, becomes the reason for neutrophils migration in the forming infiltrate [28].

It is not proven yet if $B$. burgdorferi specifically inhibits neutrophils infiltration. Proteins of the tick's saliva have an inhibiting influence on the adherence and ability to kill. Despite the low number of neutrophils in the skin lesion, they show a considerable expression of HLADR and CD14 [29]. The role of DCs is phagocytosis of the pathogen cell, transformation and presentation of the bacterial antigens, which lead to the activation of specific T lymphocytes. Phagocytic abilities of DCs are differentiated. Dendritic cells skin cells have higher ability of internalization of $B$. burgdorferi than Langerhans cells of the epidermis. Filgueira et al. showed that over $50 \%$ of DCs absorbed the pathogen, whereas it was $<5 \%$ in the case of Langerhans cells [30].

Salazar et al. observed a 5-fold increase in the DCs in the area of EM. The panel of markers was used to perform further characteristics of the B. burgdorferi infection influence on DCs and the assessment of CD11c surface expression, what helped to define DCs subgroups of the monocyte and lymphoid precursors. In the case of B. burgdorferi infections, there were no statistically significant differences in the CD11c+ percentage (monocytoid) and CD11c- (plasmacytoid) DCs in the peripheral blood in relation to the aspirates from the skin lesion. It indicates that none of the DCs subgroups was selectively recruited in the place of contact with the spirochaete, however there were significant differences in the activation and maturation of DCs stadia in both compartments. It was mostly visible in the case of $\mathrm{CD} 11 \mathrm{c}+$ in the percentage and the value of mean fluorescence intensity (MFI). A significant increase in CD83+ and CD11c- within skin infiltration was observed. CD1a expression suggested monocytoid DCs with the ability to differentiation into Langerhans cells. The lack of CD1a in CD11c- DCs, which stem from the infected skin, was compatible with their putative lymphoid origin [29].

A subpopulation of DCs Cd11c+/CD1a in the skin lesion, which most likely is composed of sedentary epidermal Langerhans cells plays an important role in the pathogenesis of LD. In both, an early and late skin form of the disease, there are Langerhans cells constituting DCs group. Many authors have observed that the number of these cells is higher, especially in ACA in relation to the healthy skin. However the expression of MHC II is lower in EM and ACA in comparison to the healthy skin [31]. It could explain the increase in the number of DCs in both lesions. Therefore, it could be assumed that the decrease in the MHC II synthesis indicates low effectiveness of antibacterial Th1 immunological response and that it may be partially responsible for the insufficient elimination of the pathogen isolated from ACA.

However, it is known that protective immune response is antibody dependent, but presence of MHC II molecules on DCs is not always required and other mechanisms are also possible [32]. 


\section{Toll-like receptors and Borrelia burgdorferi}

Borrelia burgdorferi has several potentially proinflammatory lipoproteins on its surface. Bacterial lipoproteins via interaction with Toll like receptors (TLRs) activate cells of the immunological system [9, 29, 33, 34, 35]. Potent proinflammatory properties of the lipoproteins were described for the first time with relation to highly immunogenic surface protein A and B and they were used to initiate the attempts to invent vaccinations against Lyme disease. So far, there have been ten TLRs types distinguished. They are characterized with the presence of 3 domains: external (ectodomain), single transmembrane and intracytoplasmic, which altogether participate in the signal transmission. The intracytoplasmic one is called TIR domain (Toll/interleukin 1 receptor homology domain) as it is homologic to the receptor for IL-1, the so-called IL-1rI. Lipoproteins of B. burgdorferi may influence TLRs, especially TLR2 receptor in the form of dimer with TLR1 or TLR6 and therefore stimulate the production of proinflammatory factors. Moreover, proteins of flagellin are recognized by TLR5 [7]. Lipoproteins are able to induce directly so-called protein complex NF- $\kappa \mathrm{B}$, which acts as a transcription factor and plays a crucial role in the regulation of the immunological response via cytokines synthesis, adhesive particles, nitrous oxygen, peroxides, etc. TLR2 and TLR4 presence on the surface of DCs participate in the recognition of lipoproteins and lipopolysaccharides. They also induce $\mathrm{NF}-\kappa \mathrm{B}$ or protein 1 activator which all belong to the transcription factors. Dendritic cells after stimulation by TLR DCs usually direct T-cells differentiation into Th1. It is assumed that innate response of restricted pathogen elimination activity and more effective protection is supported by the activation of the so-called adaptive immunological response, which stems from the secondary lymphatic organs with DCs. Lipoproteins of the spirochaete may also engage receptors other than TLRs to provoke inflammation $[36,37]$.

Toll like receptors are a group of receptors present in the host's cells such as DCs, macrophages, neutrophils or microglial cells. They constitute the first stage of defense. These receptors are also present on the T and B lymphocytes, which are responsible for the cooperation of the innate and adaptive immunological response. Wang et al. on mice models proved that stimulation of TLR2 is not only important in the antibacterial activity, but it also significantly influences pathophysiology and development of the disease as the higher number of spirochaete in the mice with TLR deficiency developed milder organ complications [38].

Another important, recently considered in the literature, issue is the function of $\gamma \delta \mathrm{T}$ cells. They accumulate at sites of inflammation in infections and autoimmune disorders, but their role is still a matter of controversy. Collins et al. observed that $\gamma \delta$ T cells in vitro are activated by
B. burgdorferi in a TLR2-dependent manner [39]. Further, Shi et al. observed that the activated $\gamma \delta$ T cells can in turn stimulate DCs in vitro to produce cytokines and chemokines that are important for the adaptive immune response. This suggested that in vivo $\gamma \delta \mathrm{T}$ cells may assist in activating the adaptive immune response. Moreover, they observed that $\gamma \delta$ T cells are activated and expand in number during $B$. burgdorferi infection, and this was reduced in the absence of TLR2. Furthermore, in the absence of $\gamma \delta \mathrm{T}$ cells, there was a significantly blunted response of adaptive immunity, as reflected in reduced expansion of $\mathrm{T}$ and $\mathrm{B}$ cells and reduced serum levels of anti-Borrelia antibodies, cytokines, and chemokines. It shows that $\gamma \delta \mathrm{T}$ cells promote the adaptive immune response during infection [40].

There has been an attempt to define the influence of B. burgdorferi infection on the surface expression of TLR receptors [29]. Peripheral CD11c+ DCs presented a higher increase in the TLR2 and TLR4 expression. Even a significantly higher TLR1, 2 and 4 expression was visible in the cells of skin lesion in the course of EM in comparison with their equivalents in the peripheral blood. It should be emphasized that TLR1 and TLR2 expression in CD11c+ in the skin lesion was higher in comparison to healthy people. The decrease in their number during the convalescence period additionally supported possible $B$. burgdorferi influence on them during the infection. Similar changes concerning TLR (monocytes, neutrophils, T lymphocytes) apply also to other cells. Moreover, B. burgdorferi lipoproteins possess potential mitogenic abilities towards B lymphocytes and are able to stimulate polyclonal activation, proliferation and production of immunoglobulins in vitro [22]. An increased number in spirochaete in mice with TLR2 deficiency is not a result of improper humoral immunological response as the production of spirochaete antibodies was normal. Such observation suggests that bacteria elimination not only requires specific antibodies, but also cells with TLR receptors.

\section{Inflammatory mediators}

In order to define changes concerning inflammatory receptors Sjowall et al. compared cytokines secretion in patients with spirochaete with the history of borreliosis to seronegative people [41]. Induction of IL-4, IL-10, IL-12p70, IFN- $\gamma$ and TNF- $\alpha$ secretion by DCs in both groups was assessed. However, the concentration of TNF- $\alpha$ turned out to be very high in a group of asymptomatic seropositive patients in comparison with the seronegative ones. There were no differences in the TNF- $\alpha$ concentration between patients with neuroborreliosis and asymptomatic patients. This is in accordance with the actual knowledge concerning the TNF- $\alpha$ participation in the elimination of the bacteria in the early phase of the disease. It could be assumed that patients with borreliosis may have 
lower ability to generate immunological response, which leads to the asymptomatic chronic inflammation and development of late forms e.g. ACA [41]. Moreover, a higher level of the IL-12p70 was observed in asymptomatic patients. Both TNF- $\alpha$ and IL-12 have essential proinflammatory and antibacterial properties. Interleukin 12 influences the induction of the proinflammatory immunological response on the Th1 lymphocytes pathway. This mechanism could be responsible for the favorable prognosis of the disease, overwhelming the antagonist anti-inflammatory response on the Th2 pathway mainly with IL-10. In the case of early stage of the disease, the dominant cytokines isolated from the lesion are IL-6 and IFN- $\gamma$ [29]. The analysis of the cytokines in serum also showed an increase in IFN- $\gamma$, TNF- $\alpha$ and IL- 2 concentrations, especially in patients with multiple erythema. Most probably not only DCs, but also other inflammatory cells take part in the increase in these mediators.

\section{Central nervous system and dendritic cells}

The central nervous system (CNS) is immunologically privileged. It has been assumed that CNS is deprived of DCs. However, this assumption has recently been verified [22]. Cells of the brain and spinal cord are almost deprived of DCs. Meninges, vascular plexus and cerebrospinal fluid (CSF) are abundant of DCs. They constitute a group of cells sufficient for the induction of T-cell response against foreign antigens. In the case of neuroborreliosis the increase in the number of MDCs and pDCs in the CSF was observed. An initial DCs increase in CSF could have been observed until two weeks. Marrow DCs in CSF presented a higher HLA-DR, CD86, CD80, CD40 expression in relation to blood, whereas the level of expression of these particles in pDCs in both, CSF and blood was the same. This suggests that MDCs have a high ability of antigen presentation and Tymphocyte activation even though the proportion of their amount in the CSF in relation to blood is lower. Plasmacytoid DCs are considered immature and called precursor cells. They have poor abilities of antigen presentation, however produce large amounts of type I IFN as a response to the infectious pathogen [22].

Factors soluble in CSF may influence the phenotype and DCs functions, which may further influence the type of T-cell intrameningeal response. Pashenkov et al. performed the incubation of immature monocyte derived DCs from healthy donors (moDC) with CSF from people with non-inflammatory neurological diseases, with sclerosis multiplex, bacterial meningitis and meningoencephalitis in the course of borreliosis. They observed that incubation of moDC with CSF with IFN- $\gamma$ in the course of borreliosis stimulates them to the increased IL-2 production and therefore the induction of potent Th-1-dependent response. In the case of CSF in the course of bacterial meningoencephalitis DCs remained immature or were transforming into macrophage like CD14+, which were relatively weaker in the induction of T-cell response in vitro [42]. This phenomenon could be explained by the significant increase in IL-10, which antagonizes the effect of IFN- $\gamma$ influence on moDC.

\section{Borrelia burgdorferi versus bacteria with lipopolysaccharide}

Hartiala et al. compared the transcription response of the human DCs to the stimulation by B. burgdorferi in relation to the one activated with lipopolysaccharide (LPS) of Escherichia coli [37]. It has been observed that the response induced by $B$. burgdorferi involves a lower number of genes in comparison with the number initiated by the bacteria with LPS. Moreover, there was a significantly higher level of CD38 in the presence of LPS. In the case of $B$. burgdorferi there was no influence on the quality of the protein. At the same time the intensity of expression of the gene coding CCR7 was examined. Analogically to CD38 LPS stimulation increased the expression of CCR7 (11-fold) more than in the case of B. burgdorferi CD38 is an ectoenzyme playing an important role in chemotaxis and migration of DC to the lymph nodes, synthesized by lymphoid cells. Its amount is low during the differentiation of immature DCs and increases at the time of maturation. CCR7 is a chemokine receptor and the main mediator in the mobilization of DC to lymph nodes. There was no significant change in the cytokine production profile in the study. CD38 and CCR7 insufficiency in the DCs caused by the inhibition of the synthesis by the spirochaete predisposes to their inability to migrate from the skin to lymph nodes after the antigen stimulation and therefore the induction of potent humoral response, which may influence further immunological reaction in the course of LD [37].

\section{Conclusions}

Dendritic cells constitute the main group of cells presenting antigens and belong to the most important step of defense against $B$. burgdorferi. As a consequence, the specific immunological response is developed in the course of Lyme disease [43].

We conclude that considering a complex role of DCs in defense mechanisms and initiation process of direct immunological response in Borrelia infection, their role should be accounted in production of vaccination against B. burgdorferi.

\section{References}

1. Nadelman RB, Wormser GP (1998): Lyme borreliosis. Lancet 352: 557-565

2. Jakóbisiak M, Romaniuk A. Dendritic cells. In: Jakóbisiak M. Immunology. PWN, Warsaw 2000; 232-234. 
3. Hovius JW (2009): Spitting image: tick saliva assists the causative agent of Lyme disease in evading host skin's innate immune response. J Invest Dermatol 129: 2337-2339.

4. Berende A, Oosting M, Kullberg BJ, et al. (2010): Activation of innate host defense mechanisms by Borrelia. Eur Cytokine Netw 21: 7-18.

5. Diebold SS. Activation of dendritic cells by Toll-like receptors and C-type lectins. In: Lombardi G, Riffo-Vasquez Y (eds.). Dendritic cells. Handbook of experimental pharmacology. Springer-Verlag, Berlin 2009; 3-30.

6. Rittig MG, Krause A, Häupl T, et al. (1992): Coiling phagocytosis is the preferential phagocytic mechanism for Borrelia burgdorferi. Infect Immun 60: 4205-4212.

7. Benach JL, Fleit HB, Habicht GS, et al. (1984): Interactions of phagocytes with the Lyme disease spirochete: role of the Fc receptor. J Infect Dis 150: 497-507.

8. Guerau-de-Arellano M, Huber BT (2005): Chemokines and Toll-like receptors in Lyme disease pathogenesis. Trends Mol Med 11: 114-120.

9. Zajkowska J, Lewczuk P, Strle F, Stanek G (2012): Lyme borreliosis: from pathogenesis to diagnosis and treatment. Clin Dev Immunol 2012: 231657.

10. Franz JK, Krause A (2003): Lyme disease (Lyme borreliosis). Best Pract Res Clin Rheumatol 17: 241-264.

11. Müllegger RR, Glatz M (2008): Skin manifestations of Lyme borreliosis: diagnosis and management. Am J Clin Dermatol 9: 355-368.

12. Hovius JW, de Jong MA, den Dunnen J, et al. (2008): Salp15 binding to DC-SIGN inhibits cytokine expression by impairing both nucleosome remodeling and mRNA stabilization. PLoS Pathog 4: e31.

13. Dai J, Wang P, Adusumilli S, et al. (2009): Antibodies against a tick protein, Salp15, protect mice from the Lyme disease agent. Cell Host Microbe 19: 482-492.

14. Sá-Nunes A, Bafica A, Antonelli LR, et al. (2009): The immunomodulatory action of sialostatin $\mathrm{L}$ on dendritic cells reveals its potential to interfere with autoimmunity. J Immunol 182: 7422-7429.

15. Vesely DL, Fish D, Shlomchik MJ, et al. (2009): Langerhans cell deficiency impairs Ixodes scapularis suppression of Th1 responses in mice. Infect Immun 77: 1881-1887.

16. Skallová A, Iezzi G, Ampenberger F, et al (2008): Development of Th2 responses maturation, and function while promoting tick saliva inhibits dendritic cell migration. J Immunol 180: 6186-6192.

17. Bonilla FA, Oettgen HC (2010): Adaptive immunity. J Allergy Clin Immunol 125: S33-40.

18. Steinman RM, Banchereau J (2007): Taking dendritic cells into medicine. Nature 449: 419-426.

19. Shen L, Rock KL (2006): Priming of T cells by exogenous antigen cross-presented on MHC class I molecules. Curr Opin Immunol 18: 85-91.

20. Villadangos JA, Heath WR, Carbone FR (2007): Outside looking in: the inner workings of the cross-presentation pathway within dendritic cells. Trends Immunol 28: 45-47.

21. Kroczek RA, Henn V (2012): The role of XCR1 and its ligand $\mathrm{XCL} 1$ in antigen cross-presentation by murine and human dendritic cells. Front Immunol 3: 14.

22. Pashenkov M, Huang YM, Kostulas V, et al. (2001): Two subsets of dendritic cells are present in human cerebrospinal fluid. Brain 124: 480-492.
23. Guilliams M, Henri S, Tamoutounour S, et al. (2010): From skin dendritic cells to a simplified classification of human and mouse dendritic cell subsets. Eur J Immunol 40: 2089-2094.

24. Bachem A, Güttler S, Hartung E, et al. (2010): Superior antigen cross-presentation and XCR1 expression define human CD11c+CD141+ cells as homologues of mouse CD8+ dendritic cells. J Exp Med 207: 1273-1281.

25. Contreras V, Urien C, Guiton R, et al. (2010): Existence of $\mathrm{CD} 8 \alpha$-like dendritic cells with a conserved functional specialization and a common molecular signature in distant mammalian species. J Immunol 185: 3313-3325.

26. Haniffa M, Shin A, Bigley V, et al. (2012): Human tissues contain CD141hi cross-presenting dendritic cells with functional homology to mouse CD103+ nonlymphoid dendritic cells. Immunity 37: 60-73.

27. Müllegger RR, McHugh G, Ruthazer R, et al. (2000): Differential expression of cytokine mRNA in skin specimens from patients with erythema migrans or acrodermatitis chronica atrophicans. J Invest Dermatol 115: 1115-1123.

28. Suhonen J, Komi J, Soukka J, et al. (2003): Interaction between Borrelia burgdorferi and immature human dendritic cells. Scand J Immunol 58: 67-75.

29. Salazar JC, Pope CD, Sellati TJ, et al. (2003): Coevolution of markers of innate and adaptive immunity in skin and peripheral blood of patients with erythema migrans. J Immunol 171: 2660-2670.

30. Filgueira L, Nestlé FO, Rittig M, et al. (1996): Human dendritic cells phagocytose and process Borrelia burgdorferi. J Immunol 157: 2998-3005.

31. Silberer M, Koszik F, Stingl G, Aberer E (2000): Downregulation of class II molecules on epidermal Langerhans cells in Lyme borreliosis. Br J Dermatol 143: 786-794.

32. Mbow ML, Zeidner N, Gilmore RD Jr, et al. (2001): Major histocompatibility complex class II-independent generation of neutralizing antibodies against T-cell-dependent Borrelia burgdorferi antigens presented by dendritic cells: regulation by NK and $\gamma \delta$ cells. Infec Immun 69: 2407-2415.

33. Bolz DD, Sundsbak RS, Ma Y, et al. (2004): MyD88 plays a unique role in host defense but not arthritis development in Lyme disease. J Immunol 173: 2003-2010.

34. Liu N, Montgomery RR, Barthold SW, Bockenstedt LK (2004): Myeloid differentiation antigen 88 deficiency impairs pathogen clearance but does not alter inflammation in Borrelia burgdorferi-infected mice. Infect Immun 72: 3195-3203.

35. Oleś D, Szczepankiewicz A (2012): Role of Toll-like receptors in the development of allergic inflammation in asthma. Postep Derm Alergol 2012; 29: 275-278.

36. Hartiala P, Hytönen J, Pelkonen J, et al. (2007): Transcriptional response of human dendritic cells to Borrelia garinii - defective CD38 and CCR7 expression detected. J Leukoc Biol 82: 33-43.

37. Hartiala P, Hytönen J, Yrjänäinen H, et al. (2010): TLR2 utlization of Borrelia does not induce $\mathrm{p} 38$ - and IFN- $\beta$ autocrine loop-dependent expression of $\mathrm{CD} 38$, resulting in poor migration and weak IL-12 secretion of dendritic cells. J Immunol 184: 5732-5742.

38. Wang X, Ma Y, Yoder A, et al. (2008): T cell infiltration is associated with increased Lyme arthritis in TLR2-/- mice. FEMS Immunol Med Microbiol 52: 124-133.

39. Collins C, Shi C, Russell JQ, et al. (2008): Activation of gamma delta $\mathrm{T}$ cells by Borrelia burgdorferi is indirect via a TLRand caspase-dependent pathway. J Immunol 15: 2392-2398. 
40. Shi C, Sahay B, Russell JQ, et al. (2011): Reduced immune response to Borrelia burgdorferi in the absence of $\gamma \delta \mathrm{T}$ cells. Infect Immun 79: 3940-3956.

41. Sjöwall J, Carlsson A, Vaarala O, et al. (2005): Innate immune responses in Lyme borreliosis: enhanced tumor necrosis factor- $\alpha$ and interleukin-12 in asymptomatic individuals in response to live spirochetes. Clin Exp Immunol 141: 89-98.

42. Pashenkov M, Söderström M, Huang YM, Link H (2002): Cerebrospinal fluid affects phenotype and functions of myeloid dendritic cells. Clin Exp Immunol 128: 379-387.

43. Schuijt TJ, Hovius JW, van der Poll T, et al. (2011): Lyme borreliosis vaccination: the facts, the challenge, the future. Trends Parasitol 27: 40-47. 